\title{
A Calculation of the Number of Lattice Points in the Circle and Sphere
}

\author{
W. Fraser and C. C. Gotlieb
}

1. Introduction. Let $A_{k}(x)$ be the number of points $\left(y_{1}, y_{2}, \cdots y_{k}\right)$ satisfying

$$
y_{1}^{2}+y_{2}^{2}+\cdots y_{k}^{2} \leqq x
$$

where $y_{1}, y_{2} \cdots y_{k}$ are integers (positive, negative or zero). Thus, $A_{k}(x)$ is the number of lattice points in a $k$-dimensional hypersphere of radius $x^{1 / 2}$. This paper describes the calculation of a table of $A_{2}(x)$ and $A_{3}(x)$ on an IBM 650 computer.

As a first approximation these are, respectively, the area and volume of the circle and sphere, but the question is how good these approximations are. In general, we are interested in

$$
P_{k}(x)=A_{k}(x)-V_{k}(x)
$$

where the volume of a sphere of radius $x^{1 / 2}$ in $k$-dimensional hyperspace is

$$
V(x)=\frac{\Pi^{k / 2} x^{k / 2}}{\Gamma(k / 2+1)} .
$$

$P_{2}(x)$ has been investigated by many celebrated mathematicians and Wilton [1] gives an account of the early work. More recently there have been theoretical investigations of $P_{k}(x)$ for higher dimensions, particularly by Walfisz [2], whose notation is being followed here.

We write $P_{2}(x)=\mathrm{O}\left(x^{c}\right)$ to mean, in the usual sense, that there exists $K$ such that

$$
\begin{array}{cl}
\left|P_{2}(x)\right| / x^{c} \leqq K & \text { as } x \rightarrow \infty, \text { and } \\
P_{2}(x)=\mathrm{o}\left(x^{c}\right) & \text { to mean that } \\
P_{2}(x) / x^{c} \rightarrow 0 & \text { as } x \rightarrow \infty .
\end{array}
$$

Further, after Littlewood, we write $P_{2}(x)=\Omega\left(x^{c}\right)$ to mean that there exists $K>0$, and a sequence of values of $x$ tending to infinity, for which

$$
\left|P_{2}(x)\right| / x^{c} \geqq K,
$$

that is, the negation of $P_{2}(x)=o\left(x^{c}\right)$. Gauss observed that

$$
P_{2}(x)=\mathrm{O}\left(x^{1 / 2}\right)
$$

Hua [3] has shown that

$$
P_{2}(x)=\mathrm{O}\left(x^{13 / 40}\right)
$$

and Van der Corput [4] that

$$
P_{2}(x)=\mathrm{o}\left(x^{1 / 3}\right)
$$

Received August 7, 1961. 
TABLE 1

\begin{tabular}{|c|c|c|c|c|c|}
\hline & Contribution & to $A_{3}(x)-F i r s t$ & Method & & \\
\hline \multirow{2}{*}{ Name } & \multirow{2}{*}{ Solutions for: } & \multirow{2}{*}{ Conditions } & \multicolumn{2}{|c|}{$\begin{array}{c}\text { Symmetry } \\
\text { Factors }\end{array}$} & \multirow{2}{*}{$\begin{array}{l}\text { Multi- } \\
\text { plicity }\end{array}$} \\
\hline & & & $\begin{array}{c}\text { Permu- } \\
\text { tation }\end{array}$ & $\begin{array}{c}\text { Reflec- } \\
\text { tion }\end{array}$ & \\
\hline $\begin{array}{l}T \\
U \\
V \\
W \\
S \\
Y \\
Z\end{array}$ & $\begin{array}{c}y_{1}{ }^{2}+y_{2}{ }^{2}+y_{3}{ }^{2} \leqq x \\
y_{1}{ }^{2}+y_{2}{ }^{2} \leqq x \\
2 y_{1}{ }^{2}+y_{2}{ }^{2} \leqq x \\
y_{1}{ }^{2}+2 y_{2}{ }^{2} \leqq x \\
y_{1}{ }^{2} \leqq x \\
2 y_{1}{ }^{2} \leqq x \\
3 y_{1}{ }^{2} \leqq x\end{array}$ & $\begin{aligned} & y_{1}>y_{2}>y_{3}>0 \\
& y_{1}>y_{2}>0 \\
& y_{1}>y_{2}>0 \\
& y_{1}>y_{2}>0 \\
& \\
& y_{1}>0 \\
& y_{1}>0 \\
& y_{1}>0\end{aligned}$ & $\begin{array}{l}6 \\
6 \\
3 \\
3 \\
3 \\
3 \\
1\end{array}$ & $\begin{array}{l}8 \\
4 \\
8 \\
8 \\
2 \\
4 \\
8\end{array}$ & $\begin{array}{r}48 \\
24 \\
24 \\
24 \\
6 \\
12 \\
8\end{array}$ \\
\hline
\end{tabular}

Hardy has shown that

$$
P_{2}(x)=\Omega\left(x^{1 / 4}\right),
$$

so that we may write

$$
P_{2}(x)=\mathrm{O}\left(x^{c}\right)
$$

where $c \geqq \frac{1}{4}$ and the best value is known to be less than $13 / 40$. It is considered probable that $c$ is arbitrarily close to $\frac{1}{4}$. Besides furnishing numerical values of $A_{2}(x)$ and $A_{3}(x)$, one purpose of this tabulation is to examine the consistency of the observed numbers with this conjecture.

Although analytic number theory yields considerable information on the behavior of $P_{k}(x)$ for $k \geqq 4$, there seems to be little reported on $P_{3}(x)$. In general

$$
P_{k}(x)=\mathrm{O}\left(x^{(k-1) / 2}\right) \text { and } P_{k}(x)=\Omega\left(x^{k / 2-1}\right)
$$

so that

$$
P_{3}(x)=\mathrm{O}\left(x^{c}\right)
$$

where it is known that $c \geqq \frac{1}{2}$ and the best value is equal to or less than 1 . These limits, of course, are not very sharp; for $P_{2}(x)$ we get from (4) only that $\frac{1}{2} \geqq c \geqq 0$. Thus, another purpose of the tabulation is to obtain some information about the behavior of $P_{3}(x)$.

2. Computing Formulas. There are many summation formulas which can be derived for $A_{3}(x)$. Essentially they are all modified enumerations where advantage is taken of the symmetries present.

Table 1 shows a decomposition into the terms which contribute to $A_{3}(x)$. Allowing for the solution at the origin, we then get

$$
A_{3}(x)=48 T+24(U+V+W)+6 S+12 Y+8 Z+1^{*}
$$

* As noted by Legendre this same result is reached by noting that $A_{3}(x)$ is given by.the number of terms having coefficients $\leqq x$ in the expansion of $\left(1+2 \Sigma_{i} y^{i^{2}}\right)\left(1+2 \Sigma_{i} y^{j^{2}}\right)$ $\left(1+2 \Sigma_{k} y^{k^{2}}\right)$. 
We proceed to find expressions for each of these terms. Let $[\sqrt{N}]$ be the largest integer equal to or less than the square root of $N$, i.e.,

$$
[\sqrt{N}] \leqq \sqrt{N}<[\sqrt{N}]+1 \text {. }
$$

Then

$$
S=[\sqrt{x}] ; \quad Y=[\sqrt{x / 2}] ; \quad Z=[\sqrt{x / 3}] .
$$

The other terms in (5) are evaluated by summation formulas. Consider the number of solutions of

$$
y_{1}^{2}+y_{2}^{2} \leqq x \text { with } y_{1} \neq 0, \quad y_{2} \neq 0
$$

For each $y_{1}$, the number of permitted values of $y_{2}$ is $\left[\sqrt{x-y_{1}^{2}}\right]$, so that the number of solutions of $(6)$ is given by $\left.\sum_{\substack{1 \sqrt{x}] \\ y_{1}=1}}^{\left[\sqrt{x-y_{1}^{2}}\right.}\right]$. But this is equal to

That is,

$$
\begin{aligned}
& \text { the number of solutions with } y_{1}>y_{2}>0 \\
& + \text { the number of solutions with } y_{2}>y_{1}>0 \\
& + \text { the number of solutions with } y_{1}=y_{2} .
\end{aligned}
$$

$$
2 U+Y=\sum_{y_{1}=1}^{[\sqrt{x}]}\left[\sqrt{x-y_{1}^{2}}\right] .
$$

A similar argument shows that

$$
V+W=\left[\sum_{y_{1}=1}^{\left[\sqrt{x_{12}}\right]}\left[\sqrt{x-2 y_{1}^{2}}\right]-[\sqrt{x / 3}]\right.
$$

again, we have the number of solutions of

$$
\begin{aligned}
y_{1}{ }^{2}+y_{2}{ }^{2}+y_{3}^{2} \leqq x \text { for } y_{1}>y_{2}>0=3 T+V & +w \\
& =\sum_{y_{1}=2}^{[\sqrt{x-1}]} \sum_{y_{2}=1}^{\min }\left[\sqrt{x-y_{1}{ }^{2}-y_{2}^{2}}\right]
\end{aligned}
$$

where $\min$ is the lesser of $\left(y_{1}-1\right)$ and $\left[\sqrt{x-y_{1}^{2}}\right]$. Thus we finally get

$$
\begin{aligned}
A_{3}(x)=16 \sum_{y_{1}=1}^{[\sqrt{x-1}]} \sum_{y_{2}=1}^{\min }\left[\sqrt{x-y_{1}^{2}-y_{2}^{2}}\right]+8 \sum_{y_{1}=1}^{\left[\sqrt{x_{12}}\right]}\left[\sqrt{x-2 y_{1}^{2}}\right] \\
+12 \sum_{y_{1}=1}^{[\sqrt{x}]}\left[\sqrt{x-y_{1}^{2}}\right]+6[\sqrt{x}]+1
\end{aligned}
$$

Further, it is easily seen that

$$
A_{2}(x)=4 \sum_{y_{1}=1}^{[\sqrt{x}]}\left[\sqrt{x-y_{1}^{2}}\right]+4[\sqrt{x}]+1 .
$$

These equations, (7) and (8), were the two actually used the first time the table was computed. If it had been practical to compute in successive values of $x$ it probably would have been best to use a difference formula to find the contributions from successive spherical shells. Actually it was decided to compute in equal intervals 
of $x^{1 / 2}$ for a while, and then to skip to large arguments so that the asymptotic values could be examined. The IBM 650 computer used for the calculation was equipped with core storage and index registers. Although the full program required only 168 instructions and the inner loop was completely in the 60 -word core storage, it took about 10 hours to compute $A_{3}\left(10^{6}\right)$, the largest value for which equation (7) was used.

A second and independent program was written for computing $A_{2}(x)$ and $A_{3}(x)$. This program was based on the equations:

$$
\begin{aligned}
A_{3}(x)= & 1+6[\sqrt{x}]+12\left[\sqrt{\frac{x}{2}}\right]+8\left[\sqrt{\frac{x}{3}}\right]+24 \sum_{y_{1}=1}^{L_{1}}\left(\left[\sqrt{x-y_{1}^{2}}\right]-y_{1}\right) \\
& +24 \sum_{y_{1}=1}^{L_{2}}\left(\left[\sqrt{x-2 y_{1}^{2}}\right]-y_{1}\right)+24 \sum_{y_{1}=1}^{L_{3}}\left(\left[\sqrt{\frac{x-y_{1}^{2}}{2}}\right]-y_{1}\right) \\
& +48 \sum_{y_{1}=1}^{L_{4}} \sum_{y_{2}=y_{1}+1}^{L_{5}}\left(\left[\sqrt{x-y_{1}^{2}-y_{2}^{2}}\right]-y_{2}\right)
\end{aligned}
$$

where

$L_{1}$ is the largest integer, $y_{1}$ satisfying $y_{1}{ }^{2}+\left(y_{1}+1\right)^{2} \leqq x$

$L_{2}$ is the largest integer, $y_{1}$ satisfying $2 y_{1}{ }^{2}+\left(y_{1}+1\right)^{2} \leqq x$

$L_{3}$ is the largest integer, $y_{1}$ satisfying $y_{1}{ }^{2}+2\left(y_{1}+1\right)^{2} \leqq x$

$L_{4}$ is the largest integer, $y_{1}$ satisfying $y_{1}{ }^{2}+\left(y_{1}+1\right)^{2}+\left(y_{1}+2\right)^{2} \leqq x$

$L_{5}$ is the largest integer, $y_{2}\left(y_{1}\right)$ satisfying $y_{1}{ }^{2}+y_{2}{ }^{2}+\left(y_{1}+1\right)^{2} \leqq x$;

$$
A_{2}(x)=1+4[\sqrt{x}]+4[\sqrt{x / 2}]+8 \sum_{y_{1}=1}^{L_{0}}\left(\left[\sqrt{x-y_{1}^{2}}\right]-y_{1}\right)
$$

where $L_{0}$ is the largest integer, $y_{1}$ satisfying $y_{1}{ }^{2}+\left(y_{1}+1\right)^{2} \leqq x$. These equations can be obtained by counting the lattice points according to the scheme illustrated in Figure 1 and Table 2.

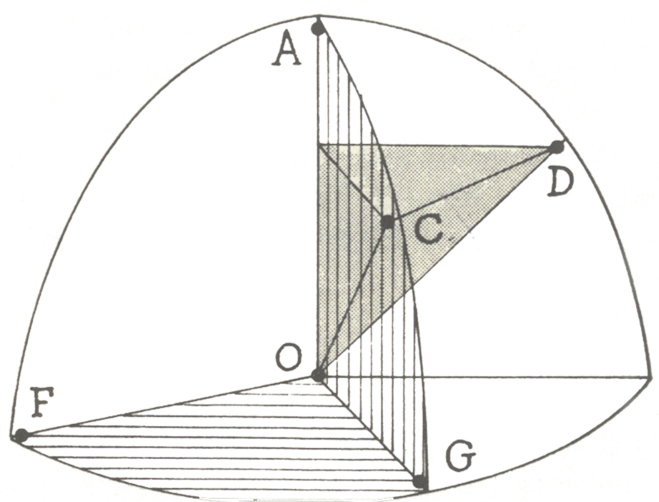

FIG. 1. Decomposition of $A_{3}(x)$-second method. 
TABLE 2

\begin{tabular}{l|c|c}
\multicolumn{3}{|c}{ Contributions to $A_{3}(x)$-Second Method } \\
\hline Source & Multiplicity & Number of Points \\
\hline Point O & 1 & 1 \\
Line OF & 6 & {$[\sqrt{x}]$} \\
Line OG & 12 & {$[\sqrt{x / 2}]$} \\
Line OC & 8 & {$[\sqrt{x / 3}]$} \\
Plane OFG & 24 & $\sum\left(\left[\sqrt{x-y_{1}^{2}}\right]-y_{1}\right)$ \\
Plane OCA & 24 & $\sum\left(\left[\sqrt{x-2 y_{1}^{2}}\right]-y_{1}\right)$ \\
Plane OCG & 24 & $\left.\sum\left(\sqrt{\frac{x-y_{1}^{2}}{2}}\right]-y_{1}\right)$ \\
Volume OACD & 48 & $\sum \sum\left(\left[\sqrt{x-y_{1}^{2}-y_{2}^{2}}\right]-y_{2}\right)$ \\
\hline
\end{tabular}

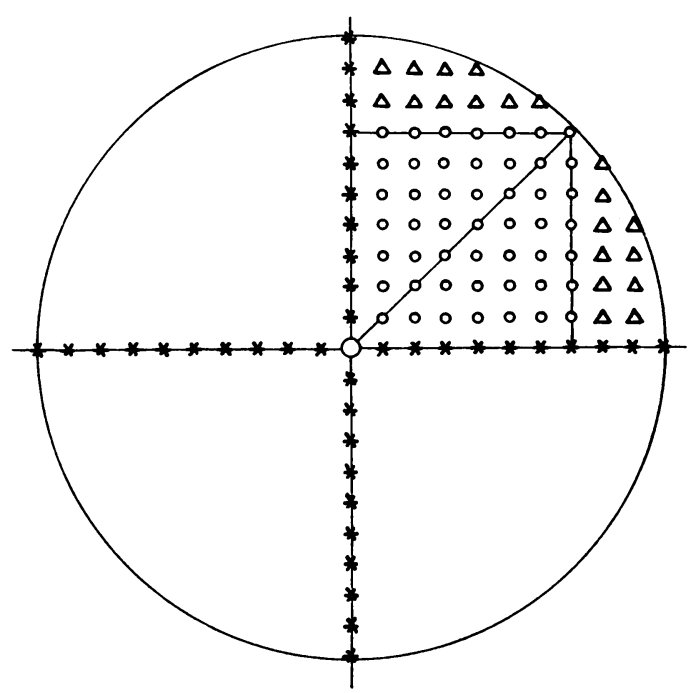

Fig. 2. Decomposition of $A_{2}(x)$-Gauss method.

This second program was faster than the first, and it was possible to extend the calculation to $x=3.24 \times 10^{6}$, which value took about $1 \frac{1}{2}$ hours to compute. For this value, $A_{3}(x)$ exceeds the 10 digits of the storage positions, but the individual sums contributing to the result are still within single-precision range.

Since the calculation was done it has been realized that there are formulas which might be even more efficient for computing. These result from the following equation, noted by Gauss:

$$
A_{2}(x)=1+4[\sqrt{x}]+4[\sqrt{x / 2}]^{2}+8 \sum_{y_{1}=[\sqrt{x / 2}]+1}^{[\sqrt{x}]}\left[\sqrt{x-y_{1}^{2}}\right] .
$$

The terms contributing to $A_{2}(x)$ are shown in Figure 2, and it is seen that equation (11) is superior to equations (8) and (10) for computing because the contribution given by $4[\sqrt{x / 2}]^{2}$ has been removed from the summation. When equation (11) 
TABLE 3

Computed Results

\begin{tabular}{|c|c|c|c|c|}
\hline$X^{1 / 2}$ & $A_{3}(X)$ & $\left|P_{3}(X)\right|$ & $A_{2}(X)$ & $\left|P_{2}(X)\right|$ \\
\hline 1 & 7 & 3 & $\tilde{\jmath}$ & 2 \\
\hline 2 & 33 & 1 & 13 & 0 \\
\hline 3 & 123 & 10 & 29 & 1 \\
\hline 4 & 257 & 11 & 49 & 1 \\
\hline 5 & 515 & 9 & 81 & 2 \\
\hline 6 & 925 & 20 & 113 & 0 \\
\hline 7 & 1419 & 18 & 149 & 5 \\
\hline 8 & 2109 & 36 & 197 & 4 \\
\hline 9 & 3071 & 17 & 253 & 1 \\
\hline 10 & 4169 & 20 & 317 & 3 \\
\hline 11 & 5575 & & 377 & 3 \\
\hline 12 & 7153 & 85 & 441 & 11 \\
\hline 13 & 9171 & 32 & 529 & 2 \\
\hline 14 & 11513 & 19 & 613 & 3 \\
\hline 15 & 14147 & 10 & 709 & 2 \\
\hline 16 & 17077 & 80 & 797 & 7 \\
\hline 17 & 20479 & 101 & 901 & 7 \\
\hline 18 & 24405 & 24 & 1009 & 9 \\
\hline 19 & 28671 & 60 & 1129 & 5 \\
\hline 20 & 33401 & 109 & 1257 & 0 \\
\hline 21 & 38911 & 119 & 1373 & 12 \\
\hline 22 & 44473 & 129 & 1517 & 4 \\
\hline 23 & 50883 & 82 & 1653 & 9 \\
\hline 24 & 57777 & 129 & 1793 & 17 \\
\hline 25 & 65267 & 183 & 1961 & 2 \\
\hline 26 & 73525 & 97 & 2121 & 3 \\
\hline 27 & 82519 & 71 & 2289 & 1 \\
\hline 28 & 91965 & 13 & 2453 & 10 \\
\hline 29 & 101943 & 217 & 2629 & 13 \\
\hline 30 & 113081 & 16 & 2821 & 6 \\
\hline 31 & 124487 & 301 & 3001 & 18 \\
\hline 32 & 137065 & 193 & 3209 & 8 \\
\hline 33 & 150555 & 22 & 3409 & 12 \\
\hline 34 & 164517 & 119 & 3625 & 7 \\
\hline 35 & 179579 & 15 & 3853 & 5 \\
\hline 36 & 195269 & 163 & 4053 & 19 \\
\hline 37 & 212095 & 80 & 4293 & 8 \\
\hline 38 & 229549 & 298 & 4513 & 23 \\
\hline 39 & 248439 & 36 & 4777 & 1 \\
\hline 40 & 267761 & 322 & 5025 & 2 \\
\hline 41 & 288359 & 337 & 5261 & 20 \\
\hline 42 & 310177 & 162 & 5525 & 17 \\
\hline 43 & 332779 & 259 & 5789 & 20 \\
\hline 44 & 356637 & 181 & 6077 & 5 \\
\hline 45 & 381915 & 211 & 6361 & 1 \\
\hline 46 & 407597 & 123 & 6625 & 23 \\
\hline 47 & 434551 & 342 & 6921 & 19 \\
\hline 48 & 462781 & 466 & 7213 & 25 \\
\hline 49 & 492567 & 240 & 7525 & 18 \\
\hline
\end{tabular}


TABLE 3-Continued

\begin{tabular}{|c|c|c|c|c|}
\hline$X^{1 / 2}$ & $A_{3}(X)$ & $\left|P_{3}(X)\right|$ & $A_{2}(X)$ & $\left|P_{2}(X)\right|$ \\
\hline 50 & 523305 & 294 & 7845 & 9 \\
\hline 55 & 696507 & 403 & 9477 & 26 \\
\hline 60 & 904089 & 690 & 11289 & 21 \\
\hline 65 & 1149651 & 696 & 13273 & 0 \\
\hline 70 & 1436385 & 370 & 15373 & 21 \\
\hline 75 & 1767063 & 83 & 17665 & 6 \\
\hline 80 & 2143641 & 1020 & 20081 & 25 \\
\hline 85 & 2571711 & 730 & 22701 & 3 \\
\hline 90 & 3053617 & 11 & 25445 & 2 \\
\hline 95 & 3590863 & 501 & 28345 & 8 \\
\hline 100 & 4187857 & 933 & 31417 & 1 \\
\hline 105 & 4849327 & 279 & 34621 & 15 \\
\hline 110 & 5574721 & 559 & 37981 & 32 \\
\hline 115 & 6370351 & 275 & 41545 & 3 \\
\hline 120 & 7236577 & 1652 & 45225 & 14 \\
\hline 125 & 8180887 & 344 & 49077 & 10 \\
\hline 130 & 9201625 & 1147 & 53077 & 16 \\
\hline 135 & 10305407 & 588 & 57209 & 47 \\
\hline 140 & 11492081 & 1959 & 61529 & 46 \\
\hline 145 & 12768503 & 1548 & 66045 & 7 \\
\hline 150 & 14137637 & 470 & 70681 & 5 \\
\hline 155 & 15598031 & 500 & 75465 & 12 \\
\hline 160 & 17155325 & 1960 & 80381 & 44 \\
\hline 165 & 18817007 & 438 & 85501 & 29 \\
\hline 170. & 20578325 & 1201 & 90785 & 7 \\
\hline 175 & 22448927 & 371 & 96209 & 2 \\
\hline 180 & 24427317 & 1707 & 101765 & 23 \\
\hline 185 & 26520663 & 1186 & 107501 & 20 \\
\hline 190 & 28729653 & 1259 & 113369 & 42 \\
\hline 195 & 31058271 & 1085 & 119433 & 26 \\
\hline 200 & 33507885 & 2437 & 125629 & 35 \\
\hline 300 & 113094545 & 2791 & 282697 & 46 \\
\hline 400 & 268077737 & 4836 & 502625 & 30 \\
\hline 500 & 523592077 & 6699 & 785349 & 49 \\
\hline 600 & 904769241 & 9443 & 1130913 & 60 \\
\hline 700 & 1436743985 & 11055 & 1539297 & 83 \\
\hline 800 & 2144654669 & 5916 & 2010573 & 46 \\
\hline 900 & 3053616505 & 11554 & 2544569 & 121 \\
\hline 1000 & 4188781437 & 8768 & 3141549 & 44 \\
\hline 1200 & 7238202017 & 27457 & 4523793 & 100 \\
\hline 1400 & 11494026189 & 14133 & 6157477 & 145 \\
\hline 1600 & 17157266213 & 18466 & 8042349 & 128 \\
\hline 1800 & 24428980617 & 43857 & 10178545 & 215 \\
\hline
\end{tabular}

is integrated plane-by-plane there results

$$
\begin{aligned}
& A_{3}(x)=1+6[\sqrt{x}]+12[\sqrt{x / 2}]+24 \sum_{y_{1}=[\sqrt{x / 2}]+1}^{(\sqrt{x})}\left[\sqrt{x-y_{1}^{2}}\right] \\
& \quad+8 \sum_{y_{1}=1}^{[\sqrt{x-1}]}\left[\sqrt{\left.\frac{x-y_{1}^{2}}{2}\right]+16 \sum_{y_{1}=1}^{[\sqrt{x=1}]} \sum_{y_{2}=\left[\sqrt{\frac{x-y_{1}}{2}}\right]}\left[\sqrt{\frac{x-y_{1}{ }^{2}}{2}}+1\right]}\left[\sqrt{x-y_{1}^{2}-y_{2}^{2}}\right] .\right.
\end{aligned}
$$


Still other summation formulas can be derived and it is likely that the most efficient one will depend on the computer being used. A method of decomposition for finding $A_{k}(x)$ which is best for $k=2$ is not necessarily best for higher values of $k$.

3. Results. A partial table of computed results is shown in Table 3. Shown there are $x^{1 / 2}, A_{3}(x),\left|P_{3}(x)\right|, A_{2}(x)$ and $\left|P_{2}(x)\right|$ for the following range:

$$
x^{1 / 2}=1(1) 50(5) 200(100) 1000(200) 1800 .
$$

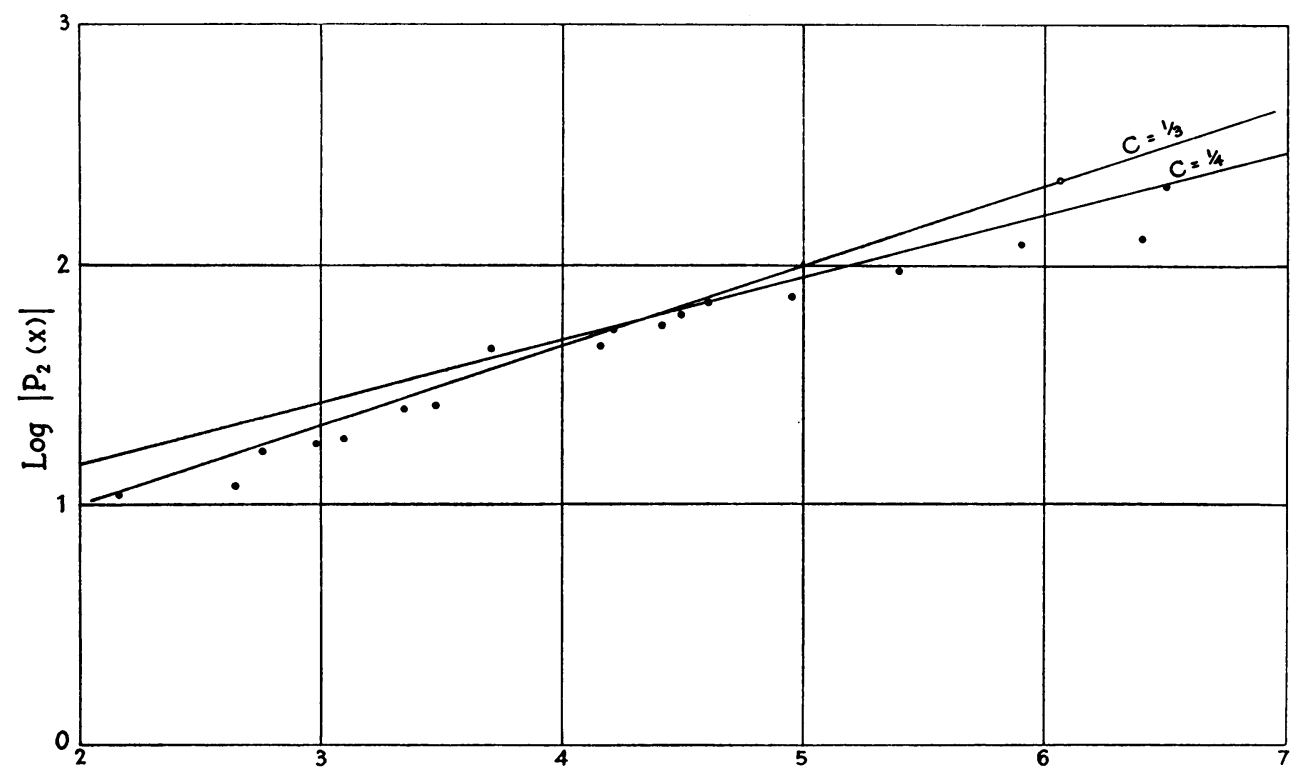

Fig. 3. $\log X$.

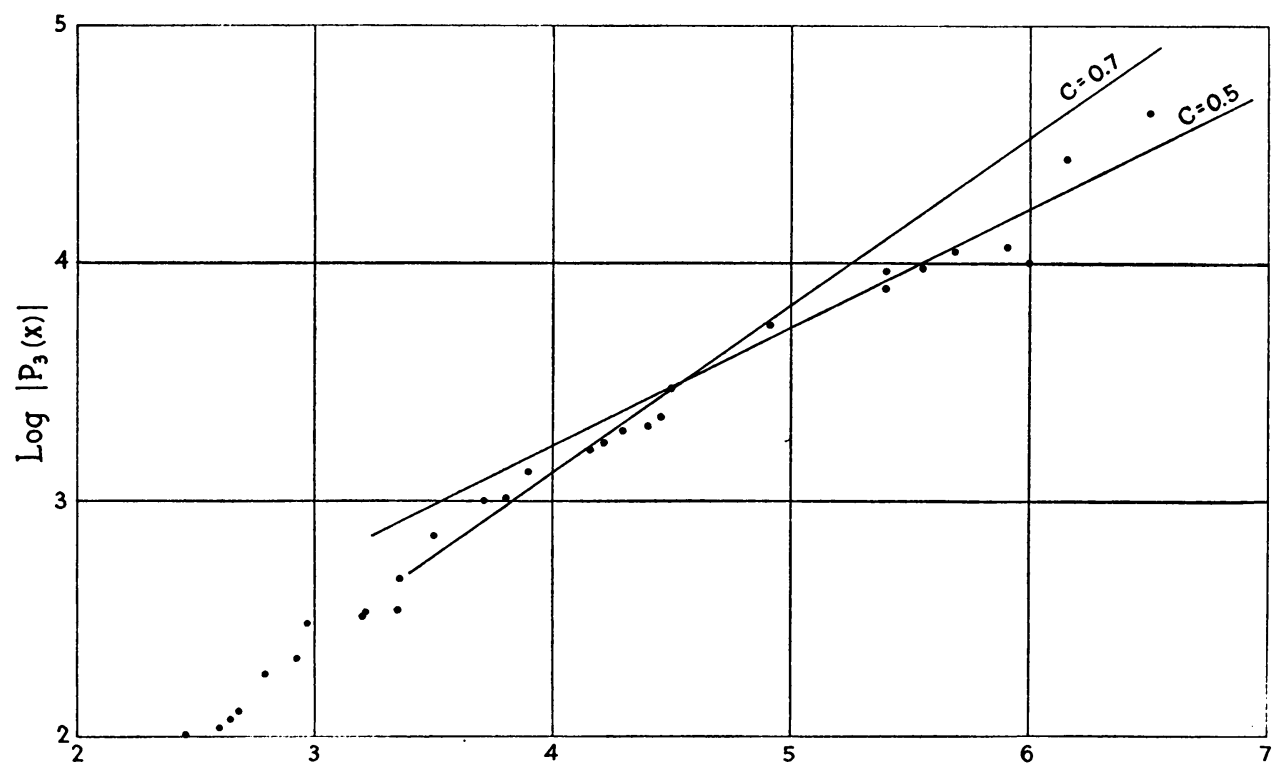

Frg. 4. $\log X$. 
All of these entries except those for $x^{1 / 2}=1200,1400,1600$ and 1800 were computed by the two independent programs. The results agree with those given in the short table of reference [5]. Calculations were actually made for about 250 arguments in the range $x^{1 / 2}=1$ to 2000 .

Because it is known that $P_{2}(x)=\Omega\left(x^{1 / 4}\right)$ the points of greatest interest in investigating the asymptotic behavior of $P_{2}(x)$ are those local maxima, $M_{i}$ of $\left|P_{2}(x)\right|$, satisfying $\left|P_{2}\left(M_{i}\right)\right|>\left|P_{2}(x)\right|$ for all $x<M_{i}$. From the tabulated results it is only possible to obtain an estimate of these $M_{i}$ and the corresponding values of $\left|P_{2}\left(M_{i}\right)\right|$.

Figure 3 shows a graph of $\log \left|P_{2}(x)\right|$ versus $\log x$ for those computed values of $x$ where $\log \left|P_{2}(x)\right|$ is larger than any preceding value. We have drawn two lines on this graph, one with slope $1 / 3$, and the other with slope $1 / 4$. The line with slope $1 / 3$ looks too steep, that is, one feels that the points will continue to lie more and more below the line. The line with slope $1 / 4$ looks reasonable, from which one can conclude that the conjecture that $c$ is arbitrarily close to $1 / 4$ is not inconsistent with the observed results. However some unpublished computations by Harry Mitchell of the Lockheed Missiles and Space Corporation show that for some $x$ between $10^{6}$ and $10^{10}$ the values of $\left|P_{2}(x)\right| / x^{1 / 4}$ grow very remarkably.

Figure 4 shows a graph of $\log \left|P_{3}(x)\right|$ versus $\log x$, for those computed values of $x$ where $\log \left|P_{3}(x)\right|$ is larger than any preceding value. Two lines, with slopes 0.5 and 0.7 have been drawn. It looks as if the points will, in the main, continue to lie between these lines, from which we conjecture that

$$
\left|P_{3}(x)\right|=\mathrm{O}\left(x^{c}\right)
$$

where $0.5 \leqq c \leqq 0.7$.

We would like to thank D. B. Scott, University of Toronto, whose investigations into close packing of spheres first suggested this calculation, and G. Forsythe, Stanford University, with whom we have had helpful discussions.

Defence Research Board

University of Toronto

Computation Center

University of Toronto

1. J. R. Wilton, "The lattice points of a cirlce: an historical account of the problem," Messenger of Mathematics, v. 58, 1929, p. 67-80.

2. A. WALFIsz, Gitterpunkte in Mehrdimensionalen Kugeln, Monografie Matematyczne, v. 33, Panstwowe Wydawnictwo Naukowe, Warsaw, 1957. (Reviewed in Mathematical Reviews, v. 20 , n. 6,1959 , p. 3826 .) $18-29$.

3. L. K. HUA, "The lattice-points in a circle," Quart. J. Math., Oxford Ser. 13, 1942, p.

4. E. LANDAU, Vorlesungen üeber Zahlentheorie, Von S. Hirzel, Leipzig, 1927, p. 264.

5. Hilbert \& Cohn-Vossen, Anschauliche Geometrie, Dover, New York, 1944. p. 29. 